\title{
List of Reviewers
}

ACM ToMPECS thanks the following people, who have reviewed at least one manuscript for the journal over the past year:

Amine Abid
Eitan Altman
Christos Antonopoulos
Ognjen Arandjelovic
Krste Asanovic
Alberto Avritzer
Roberto Baldoni
Michael Beck
Daniel Berger
Luzheng Bi
David Black-Schaffer
Alessio Botta
Loc Bui
Jun Cai
Giuliano Casale
Xuhao Chen
Lucy Cherkasova
Hyungwon Choi
Dieter Claeys
Jasper de Bock
Hermann de Meer
Koen de Turck
Jan-Pieter Dorsman
Parijat Dube
Yajuan Du
Alexandra Fedorova
Giuliana Franceschinis
Daisuke Fukuda
Nicolas Gast
Ke Guan
Yanfei Guo
Abhishek Gupta
Insu Han
Danping He
Jane Hillston
Sheng-Wei Huang
Kai Hwang

Bo $\mathrm{Ai}$

Ganesh Ananthanarayanan

Alessandro Antonucci

Danilo Ardagna

David Atienza

Abdel-Hameed Badawy

Siddhartha Banarjee

Ahmed Bendahmane

Marko Bertogna

Robert Birke

Sergey Blagodurov

Raouf Boutaba

Rajkumar Buyya

Rodrigo Calheiros

Xiang Cheng

Yuan Chen

Bruce Childers

Andrzej Chydzinski

Italo Cunha

Gert de Cooman

Arnoud den Boer

Matthias Diener

Tien Do

Nick Duffield

Atilla Eryilmaz

Liu Feng

Jeff Frolik

Luoyi Fu

Zhiguo Ge

Fabrice Guillemin

Zhenhua Guo

Varun Gupta

Refael Hassin

Nidhi Hegde

Jan Himmelspach

Yihua Huang

Esa Hyytia
David Aikema

Ahmad Anbar

Nelson Antunes

Patricia Arias-Cabarcos

Frank Aurzada

Jose Baiocchi

Vasic Bane

Shajulin Benedict

Cor-Paul Bezemer

Mathias Bjoerkqvist

Thomas Bonald

Philip Brown

Marco Caccamo

Niklas Carlsson

Lydia Chen

Zhen Chen

Tiberiu Chis

Florin Ciucu

Franco Davoli

Walter de Donato

Peter Desnoyers

Ioannis Dimitriou

Idilio Drago

Simon Duquennoy

Kaniz Fatema

Mamoun Filali

Richard Fujimoto

Saurabh Garg

Roberto Gioiosa

Haryadi Gunawi

Zishan Guo

Mursalin Habib

Gerhard Hasslinger

Tobias Helms

I-Hong Hou

Wen $\mathrm{Hu}$

(c) 2018 ACM 2376-3639/2018/08-ART21 $\$ 15.00$

https://doi.org/10.1145/3271430 
Ilias Iliadis

Olle Jaerv

Zhen Ming Jiang

Gauri Joshi

Karim Kanoun

Valerie Kelly

Christoph Kessler

Bara Kim

Sungchan Kim

Naoru Koizumi

Samuel Kounev

Diwakar Krishnamurthy

Thomas Kurner

Michael Laurenzano

Ho Woo Lee

Baptiste Lepers

Bin Li

Keqin Li

Feng Liu

$\mathrm{Xu}$ Liu

Yong Liu

Yong $\mathrm{Li}$

Dan Ma

Roland Malhame

Ming Mao

Manish Marwah

Stephen McCamant

Warren Meck

Daniel Menasche

Isi Mitrani

Gianstefano Monni

Philippe Navaux

Dung Viet Nguyen

Sangyoon Oh

Tajudeen Olasupo

Aziz Ouaarab

DK Panda

Matthias Payer

Miguel Peon Quiros

Paul Pop

Lavanya Ramapantulu

Ralf Reussner

Antonio Augusto Rocha

Jerry Rolia

Ali Safari

Behrooz Sangchoolie
Peter Jacko

Bo Jiang

Bo Ji

Guillaume Jourjon

Daniel Karapetyan

Darren Kerbyson

Gokcen Kestor

Hyesoon Kim

Taesoo Kim

Sastry Kompella

Marios Kountouris

Lars Michael Kristensen

Bo-Cheng Lai

Euiwoong Lee

Kangwook Lee

Shuangqing Liao

Chih-ping Li

Cong Liu

Jia Liu

Xunyun Liu

Zhenhua Liu

Zhaokui Li

Siva Theja Maguluri

Ruchika Malhotra

Andrea Marin

Rivalino Matias

Alistair McEwan

David Meisner

Dan Miao

Jose Maria Molina Garcia Pardo

Fabricio Murai

Giovanni Neglia

Jose Nino-Mora

Sewoong Oh

Per-Olov Ostberg

Zhonghong $\mathrm{Ou}$

John Pang

Ramtin Pedarsani

Juan Perez

Vivien Quema

Peter Reichl

Alejandro Rico

Arun Rodrigues

Dennis Rose

Marc Sanchez Artigas

Karthikeyan Sankaralingam
Bruce Jacob

Yuming Jiang

Changhee Joo

Mahmut Kandemir

Vasileios Karyotis

Yacine Kessaci

Samee Khan

Hyoseung Kim

William Knottenbelt

Ivica Kostanic

Anne Koziolek

Fernando Kuipers

Tian Lan

Hankook Lee

Emilio Leonardi

Xiaojing Liao

Dong Li

Fangming Liu

Jiajia Liu

Yang Liu

Yang Li

Pedro Garcia Lopez

Martin Maier

Renato Mancuso

Venkata Swamy Martha

Ravi Mazumdar

Suzanne McIntosh

Daniel Menasce

Ningfang Mi

Daniel Molka

Onur Mutlu

Barry Nelson

Ulrik Nyman

Jungseul Ok

Carlos Otero

Elena Pagani

Marta Patino-Martinez

Rodolfo Pellizzoni

Harry Perros

Thierry Rakotoarivelo

Jacques Resing

Alma Riska

Wouter Rogiest

Francesc Rossello

Daniel Sanchez

Muhammad Shafique 


Xuemin Shen
Manuel Silva
Gwendal Simon
Eunhye Song
Christopher Stewart
Qihang Sun
Domenico Talia
Jian Tan
Miklos Telek
Pedro Tomas
Aalap Tripathy
Konstantinos Tsakalozos
Adelinde Uhrmacher
Thiemo Voigt
Qingyang Wang
Weina Wang
David Wentzlaff
Chuan Wu
Hong Xie
Jingteng Xue
Jose Yallouz
Gang Yao
Hyunho Yeo
Chuan Yue
Haibo Zeng
Li Zhang
Zhangui Zhong

Xuemin Shen

Manuel Silva

Eunhye Song

Christopher Stewart

Qihang Sun

Domenico Talia

Jian Tan

Miklos Telek

Konstantinos Tsakalozos

Adelinde Uhrmacher

Thiemo Voigt

Qingyang Wang

Weina Wang

David Wentzlaff

an $\mathrm{Wu}$

Hong Xie

Jingteng Xue

Jose Yallouz

Gang Yao

Hyunho Yeo

Chuan Yue

Li Zhang
Ness Shroff

Alkis Simitsis

Damjan Skulj

Stelios Sotiriadis

Marc St-Hilaire

Weiqiang Sun

Lingjia Tang

Medhat Tawfeek

Nigel Thomas

Stojan Trajanovski

Trong-Tuan $\mathrm{Vu}$

Petr Tuma

Mihaela van der Schaar

Joris Walraevens

Shangguang Wang

Zhi Wang

Joerg Widmer

Yahui Wu

Wei Xie

Jun Xu

Feng Yan

Yuan Yao

Heng Yin

Haoran Yu

Fan Zhang

Xiaoxi Zhang

Enlu Zhou
Alonso Silva

Yogesh Simmhan

Yan Solihin

Per Stenstrom

Tonghoon Suk

Yin Sun

Tao Tang

Renata Teixeira

Ananta Tiwari

Liljana Trajkovic

Catia Trubiani

Bhekisipho Twala

Alex Borges Vieira

Neil Walton

Sinon Wang

Ding Wen

Bo Wu

Cathy Xia

Min Xing

Yuedong $\mathrm{Xu}$

Can-Qun Yang

Fangming Ye

Sungjoo Yoo

Bahram Zarrin

Jian Zhang

Yueping Zhang

Xiaobo Zhou 